\title{
On the existence of $0 / 1$ polytopes with high semidefinite extension complexity
}

\author{
Jop Briët · Daniel Dadush · Sebastian Pokutta
}

Received: 23 June 2013 / Accepted: 19 April 2014 / Published online: 8 May 2014

(C) Springer-Verlag Berlin Heidelberg and Mathematical Optimization Society 2014

\begin{abstract}
In Rothvoß (Math Program 142(1-2):255-268, 2013) it was shown that there exists a $0 / 1$ polytope (a polytope whose vertices are in $\{0,1\}^{n}$ ) such that any higher-dimensional polytope projecting to it must have $2^{\Omega(n)}$ facets, i.e., its linear extension complexity is exponential. The question whether there exists a $0 / 1$ polytope with high positive semidefinite extension complexity was left open. We answer this question in the affirmative by showing that there is a $0 / 1$ polytope such that any spectrahedron projecting to it must be the intersection of a semidefinite cone of dimension $2^{\Omega(n)}$ and an affine space. Our proof relies on a new technique to rescale semidefinite factorizations.
\end{abstract}

Keywords Semidefinite extended formulations - Extended formulations ·

Extension complexity

Mathematics Subject Classification 90Cxx

Sebastian Pokutta research reported in this paper was partially supported by NSF grant CMMI-1300144.

Jop Briët was supported by a Rubicon grant from the Netherlands Organisation for Scientific Research (NWO).

J. Briët · D. Dadush

Courant Institute of Mathematical Sciences,

New York University, New York, NY, USA

e-mail: jop.briet@cims.nyu.edu

D. Dadush

e-mail: dadush@cims.nyu.edu

S. Pokutta $(\varangle)$

Georgia Institute of Technology, H. Milton Stewart School

of Industrial and Systems Engineering, Atlanta, GA, USA

e-mail: sebastian.pokutta@isye.gatech.edu 


\section{Introduction}

The subject of lower bounds on the size of extended formulations has recently regained a lot of attention. This is due to several reasons. First of all, essentially all NP-Hard problems in combinatorial optimization can be expressed as linear optimization over an appropriate convex hull of integer points. Indeed, many past (erroneous) approaches for proving that $\mathrm{P}=\mathrm{NP}$ have proceeded by attempting to give polynomial sized linear extended formulations for hard convex hulls (convex hull of TSP tours, indicators of cuts in a graph, etc.). Recent breakthroughs Fiorini et al. [6], Braun et al. [3] have unconditionally ruled out such approaches for the TSP and Correlation polytope, complementing the classic result of Yannakakis [17] which gave lower bounds for symmetric extended formulations. Furthermore, even for polytopes over which optimization is in $\mathrm{P}$, it is very natural to ask what the "optimal" representation of the polytope is. From this perspective, the smallest extended formulation represents the "description complexity" of the polytope in terms of a linear or semidefinite program.

A (linear) extension of a polytope $P \subseteq \mathbb{R}^{n}$ is another polytope $Q \subseteq \mathbb{R}^{d}$, so that there exists a linear projection $\pi$ with $\pi(Q)=P$. The extension complexity of a polytope is the minimum number of facets in any of its extensions. The linear extension complexity of $P$ can be thought of as the inherent complexity of expressing $P$ with linear inequalities. Note that in many cases it is possible to save an exponential number of inequalities by writing the polytope in higher-dimensional space. Well-known examples include the regular polygon, see Ben-Tal and Nemirovski [1] and Fiorini et al. [7] or the permutahedron, see Goemans [8]. A (linear) extended formulation is simply a normalized way of expressing an extension as an intersection of the nonnegative cone with an affine space; in fact we will use these notions in an interchangeable fashion. In the seminal work of Yannakakis [16] a fundamental link between the extension complexity of a polytope and the nonnegative rank of an associated matrix, the so called slack matrix, was established and it is precisely this link that provided all known strong lower bounds. It states that the nonnegative rank of any slack matrix is equal to the extension complexity of the polytope.

As shown in Fiorini et al. [6] and Gouveia et al. [9] the above readily generalizes to semidefinite extended formulations. Let $P \subseteq \mathbb{R}^{n}$ be a polytope. Then a semidefinite extension of $P$ is a spectrahedron $Q \subseteq \mathbb{R}^{d}$ so that there exists a linear map $\pi$ with $\pi(Q)=P$. While the projection of a polyhedron is polyhedral, it is open which convex sets can be obtained as projections of spectrahedra. We can again normalize the representation by considering $Q$ as the intersection of an affine space with the cone of positive semidefinite (PSD) matrices. The semidefinite extension complexity is then defined as the smallest $r$ for which there exists an affine space such that its intersection with the cone of $r \times r$ PSD matrices projects to $P$. We thus ask for the smallest representation of $P$ as a projection of a spectrahedron. In both the linear and the semidefinite case, one can think of the extension complexity as the minimum size of the cone needed to represent $P$. Yannakakis's theorem can be generalized to this case, as was done in Fiorini et al. [6] and Gouveia et al. [9], and it asserts that the semidefinite extension complexity of a polytope is equal to the semidefinite rank (see Definition 3) of any of its slack matrices. 
An important fact in the study of extended formulations is that the encoding length of the coefficients is disregarded, i.e., we only measure the dimension of the required cone. Furthermore, a lower bound on the extension complexity of a polytope does not imply that building a separation oracle for the polytope is computationally hard. Indeed, as recently shown in Rothvoß [14], the perfect matching polytope has exponential extension complexity, while the associated separation problem (which allows us to compute min-cost perfect matchings) is in P. Thus standard complexity theoretic assumptions and limitations do not apply. In fact one of the main features of extended formulations is that they unconditionally provide lower bounds for the size of linear and semidefinite programs independent of $P$ versus $N P$.

The first natural class of polytopes with high linear extension complexity comes from the work of Rothvoß [13]. Rothvoß showed that "random" 0/1 polytopes have exponential linear extension complexity via an elegant counting argument. Given that SDP relaxations are often far more powerful than LP relaxations, an important open question is whether random $0 / 1$ polytopes also have high PSD extension complexity.

\subsection{Related work}

The basis for the study of linear and semidefinite extended formulations is the work of Yannakakis (see Yannakakis [16,17]). The existence of a 0/1 polytope with exponential extension complexity was shown in Rothvoß [13] which in turn was inspired by Shannon [15]. The first explicit example, answering a long standing open problem of Yannakakis, was provided in Fiorini et al. [6] which, together with Gouveia et al. [9], also lay the foundation for the study of extended formulations over general closed convex cones. In Fiorini et al. [6] it was also shown that there exist matrices with large nonnegative rank but small semidefinite rank, indicating that semidefinite extended formulations can be exponentially stronger than linear ones, however falling short of giving an explicit proof. They thereby separated the expressive power of linear programs from those of semidefinite programs and raised the question:

\section{Does every 0/1 polytope have an efficient semidefinite lift?}

Other related work includes Braun et al. [3], where the authors study approximate extended formulations and provide examples of spectrahedra that cannot be approximated well by linear programs with a polynomial number of inequalities as well as improvements thereof by Braverman and Moitra [4]. Faenza et al. [5] proved equivalence of extended formulations to communication complexity. Recently there has been also significant progress in terms of lower bounding the linear extension complexity of polytopes by means of information theory, see Braverman and Moitra [4] and Braun and Pokutta [2]. Similar techniques are not known for the semidefinite case.

\subsection{Contribution}

We answer the above question in the negative, i.e., we show the existence of a $0 / 1$ polytope with exponential semidefinite extension complexity. In particular, we show that the counting argument of Rothvoß [13] extends to the PSD setting. 
The main challenge when moving to the PSD setting, is that the largest value occurring in the slack matrix does not easily translate to a bound on the largest values occurring in the factorizations. Obtaining such a bound is crucial for the counting argument to carry over.

Our main technical contribution is a new rescaling technique for semidefinite factorizations of slack matrices. In particular, we show that any rank- $r$ semidefinite factorization of a slack matrix with maximum entry size $\Delta$ can be "rescaled" to a semidefinite factorization where each factor has operator norm at most $\sqrt{r \Delta}$ (see Theorem 6). Here our proof proceeds by a variational argument and relies on John's theorem on ellipsoidal approximation of convex bodies John [11]. We note that in the linear case proving such a result is far simpler, here the only required observation is that after independent nonnegative scalings of the coordinates a nonnegative vector remains nonnegative. However, one cannot in general independently scale the entries of a PSD matrix while maintaining the PSD property.

Using our rescaling lemma, the existence proof of the $0 / 1$ polytopes with high semidefinite extension complexity follows in a similar fashion to the linear case as presented in Rothvoß [13]. In addition to our main result, we show the existence of a polygon with $d$ integral vertices and semidefinite extension complexity $\Omega\left(\left(\frac{d}{\log d}\right)^{\frac{1}{4}}\right)$. The argument follows similarly to Fiorini et al. [7] adapting Rothvoß [13].

\subsection{Outline}

In Sect. 2 we provide basic results and notions. We then present the rescaling technique in Sect. 3 which is at the core of our existence proof. In Sect. 4 we establish the existence of $0 / 1$ polytopes with subexponential semidefinite extension complexity and we conclude with some final remarks in Sect. 6.

\section{Preliminaries}

Let $[n]:=\{1, \ldots, n\}$. In the following we will consider semidefinite extended formulations. We refer the interested reader to Fiorini et al. [6] and Braun et al. [3] for a broader overview and proofs.

Let $B_{2}^{n} \subseteq \mathbb{R}^{n}$ denote the $n$-dimensional Euclidean ball, and let $S^{n-1}=\partial B_{2}^{n}$ denote the Euclidean sphere in $\mathbb{R}^{n}$. We denote by $\mathbb{S}_{+}^{n}$ the set of $n \times n$ PSD matrices which form a (non-polyhedral) convex cone. Note that $M \in \mathbb{S}_{+}^{n}$ if and only if $M$ is symmetric $\left(M^{\top}=M\right)$ and

$$
x^{\top} M x \geq 0 \quad \forall x \in \mathbb{R}^{n} .
$$

Equivalently, $M \in \mathbb{S}_{+}^{n}$ iff $M$ is symmetric and has nonnegative eigenvalues. For a linear subspace $W \subseteq \mathbb{R}^{n}$, let $\operatorname{dim}(W)$ denote its dimension, $W^{\perp}$ its orthogonal complement, and $P_{W}: \mathbb{R}^{n} \rightarrow \mathbb{R}^{n}$ the orthogonal projection onto $W$. Note that as a matrix $P_{W} \in \mathbb{S}_{+}^{n}$ and $P_{W}^{2}=P_{W}$. For a matrix $A \in \mathbb{R}^{n \times m}$, let $\operatorname{Im}(A)$ denote its image or column span, and let $\operatorname{Ker}(A)$ denote its kernel. For a symmetric matrix $A \in \mathbb{R}^{n \times n}$, 
we have that $\operatorname{Im}(A)=\operatorname{Ker}(A)^{\perp}$. If $A \in \mathbb{S}_{+}^{n}$, we have that $x \in \operatorname{Ker}(A) \Leftrightarrow x^{\top} A x=0$. We define the pseudo-inverse $A^{+}$of a symmetric matrix $A$ to be the unique matrix satisfying $A^{+} A=A A^{+}=P_{W}$, where $W=\operatorname{Im}(A)$. If $A$ has spectral decomposition $A=\sum_{i=1}^{k} \lambda_{i} v_{i} v_{i}^{\top}, v_{1}, \ldots, v_{k}$ orthonormal, then $A^{+}=\sum_{i=1}^{k} \lambda_{i}^{-1} v_{i} v_{i}^{\top}$.

For matrices $A, B \in \mathbb{S}_{+}^{n}$, we have that $\operatorname{Im}(A+B)=\operatorname{Im}(A)+\operatorname{Im}(B)$ and that $\operatorname{Ker}(A+B)=\operatorname{Ker}(A) \cap \operatorname{Ker}(B)$. We denote the trace of $A \in \mathbb{S}_{+}^{n}$ by $\operatorname{Tr}[A]=\sum_{i=1}^{n} A_{i i}$. For a pair of equally-sized matrices $A, B$ we let $\langle A, B\rangle=\operatorname{Tr}\left[A^{\top} B\right]$ denote their trace inner product and let $\|A\|_{F}=\sqrt{\langle A, A\rangle}$ denote the Frobenius norm of $A$. We denote the operator norm of a matrix $M \in \mathbb{R}^{m \times n}$ by

$$
\|M\|=\sup _{\|x\|_{2}=1}\|M x\|_{2}
$$

If $M$ is square and symmetric $\left(M^{\top}=M\right)$, then $\|M\|=\sup _{\|x\|_{2}=1}\left|x^{\top} M x\right|$, in which case $\|M\|$ denotes the largest eigenvalue of $M$ in absolute value. Lastly, if $M \in \mathbb{S}_{+}^{n}$ then $\|M\|=\sup _{\|x\|_{2}=1} x^{\top} M x$ by nonnegativity of the inner expression.

For every positive integer $\ell$ and any $\ell$-tuple of matrices $\mathbf{M}=\left(M_{1}, \ldots, M_{\ell}\right)$ we define

$$
\|\mathbf{M}\|_{\infty}=\max \left\{\left\|M_{i}\right\| \mid i \in[\ell]\right\} .
$$

Definition 1 (Semidefinite extended formulation) Let $K \subseteq \mathbb{R}^{n}$ be a convex set. A semidefinite extended formulation (semidefinite $\mathrm{EF}$ ) of $K$ is a system consisting of a positive integer $r$, an index set $I$ and a set of triples $\left(a_{i}, U_{i}, b_{i}\right)_{i \in I} \subseteq \mathbb{R}^{n} \times \mathbb{S}_{+}^{r} \times \mathbb{R}$ such that

$$
K=\left\{x \in \mathbb{R}^{n} \mid \exists Y \in \mathbb{S}_{+}^{r}: a_{i}^{\top} x+\left\langle U_{i}, Y\right\rangle=b_{i} \forall i \in I\right\}
$$

The size of a semidefinite EF is the size $r$ of the positive semidefinite matrices $U_{i}$. The semidefinite extension complexity of $K$, denoted $\operatorname{xc}_{\operatorname{SDP}}(K)$, is the minimum size of a semidefinite EF of $K$.

In order to characterize the semidefinite extension complexity of a polytope $P \subseteq$ $[0,1]^{n}$ we will need the concept of a slack matrix.

Definition 2 (Slack matrix) Let $P \subseteq[0,1]^{n}$ be a polytope, $I, J$ be finite sets, $\mathcal{A}=$ $\left(a_{i}, b_{i}\right)_{i \in I} \subseteq \mathbb{R}^{n} \times \mathbb{R}$ be a set of pairs and let $\mathcal{X}=\left(x_{j}\right)_{j \in J} \subseteq \mathbb{R}^{n}$ be a set of points, such that

$$
P=\left\{x \in \mathbb{R}^{n} \mid a_{i}^{\top} x \leq b_{i} \forall i \in I\right\}=\operatorname{conv}(\mathcal{X})
$$

Then, the slack matrix of $P$ associated with $(\mathcal{A}, \mathcal{X})$ is given by $S_{i j}=b_{i}-a_{i}^{\top} x_{j}$. 
Finally, the definition of a semidefinite factorization is as follows.

Definition 3 (Semidefinite factorization) Let $I, J$ be finite sets, $S \in \mathbb{R}_{+}^{I \times J}$ be a nonnegative matrix and $r$ be a positive integer. Then, a rank- $r$ semidefinite factorization of $S$ is a set of pairs $\left(U_{i}, V^{j}\right)_{(i, j) \in I \times J} \subseteq \mathbb{S}_{+}^{r} \times \mathbb{S}_{+}^{r}$ such that

$$
S_{i j}=\left\langle U_{i}, V^{j}\right\rangle
$$

for every $(i, j) \in I \times J$. The semidefinite rank of $S$, denoted $\operatorname{rank}_{\mathrm{PSD}}(S)$, is the minimum $r$ such that there exists a rank $r$ semidefinite factorization of $S$.

Using the above notions the semidefinite extension complexity of a polytope can be characterized by the semidefinite rank of any of its slack matrices, which is a generalization of Yannakakis's factorization theorem (Yannakakis [16,17]) established in Fiorini et al. [6] and Gouveia et al. [9].

Theorem 4 (Yannakakis's Factorization Theorem for SDPs) Let $P \subseteq[0,1]^{n}$ be a polytope and $\mathcal{A}=\left(a_{i}, b_{i}\right)_{i \in I}$ and $\mathcal{X}=\left(x_{j}\right)_{j \in J}$ be as in Definition 2 . Let $S$ be the slack matrix of $P$ associated with $(\mathcal{A}, \mathcal{X})$. Then, $S$ has a rank-r semidefinite factorization if and only if $P$ has a semidefinite EF of size $r$. That is, $\operatorname{rank}_{\mathrm{PSD}}(S)=\mathrm{xc}_{S D P}(P)$.

Moreover, if $\left(U_{i}, V^{j}\right)_{(i, j) \in I \times J} \subseteq \mathbb{S}_{+}^{r} \times \mathbb{S}_{+}^{r}$ is a factorization of $S$, then

$$
P=\left\{x \in \mathbb{R}^{n} \mid \exists Y \in \mathbb{S}_{+}^{r}: a_{i}^{\top} x+\left\langle U_{i}, Y\right\rangle=b_{i} \forall i \in I\right\}
$$

and the pairs $\left(x_{j}, V^{j}\right)_{j \in J}$ satisfy $a_{i}^{\top} x_{j}+\left\langle U_{i}, V^{j}\right\rangle=b_{i}$ for every $i \in I$.

In particular, the extension complexity is independent of the choice of the slack matrix and the semidefinite rank of all slack matrices of $P$ is identical.

The following well-known theorem due to John [11] lies at the core of our rescaling argument. We state a version that is suitable for the later application. Recall that $B_{2}^{n}$ denotes the $n$-dimensional Euclidean unit ball. A probability vector is a vector $p \in \mathbb{R}_{+}^{n}$ such that $p(1)+p(2)+\cdots+p(n)=1$. For a convex set $K \subseteq \mathbb{R}^{n}$, we let aff $(K)$ denote the affine hull of $K$, the smallest affine space containing $K$. We let $\operatorname{dim}(K)$ denote the linear dimension of the affine hull of $K$. Last, we let $\operatorname{relbd}(K)$ denote the relative boundary of $K$, i.e., the topological boundary of $K$ with respect to its affine hull aff $(K)$.

Theorem 5 ([11]) Let $K \subseteq \mathbb{R}^{n}$ be a centrally symmetric convex set with $\operatorname{dim}(K)=k$. Let $T \in \mathbb{R}^{n \times k}$ be such that $E=T \cdot B_{2}^{k}=\{T x \mid\|x\| \leq 1\}$ is the smallest volume ellipsoid containing $K$. Then, there exist a finite set of points $\mathcal{Z} \subseteq \operatorname{relbd}(K) \cap \operatorname{relbd}(E)$ and a probability vector $p \in \mathbb{R}_{+}^{\mathcal{Z}}$ such that

$$
\sum_{z \in \mathcal{Z}} p(z) z z^{\top}=\frac{1}{k} T T^{\top} .
$$

For a real-valued function $f: \mathbb{R} \rightarrow \mathbb{R}$, we denote its right-sided derivative at $a \in \mathbb{R}$ by 


$$
\left.\frac{d_{+}}{d x} f\right|_{x=a}=\lim _{\varepsilon \rightarrow 0^{+}} \frac{f(a+\varepsilon)-f(a)}{\varepsilon} .
$$

We will need the following lemma; for a general theory on perturbations on linear operators we refer the reader to Katō [12].

Lemma 1 Let $r$ be a positive integer, $X \in \mathbb{S}_{+}^{r}$ be a non-zero positive semidefinite matrix. Let $\lambda_{1}=\|X\|$ and $W$ denote the $\lambda_{1}$-eigenspace of $X$. Then for $Z \in \mathbb{R}^{r \times r}$ symmetric,

$$
\left.\frac{d_{+}}{d \varepsilon}\|X+\varepsilon Z\|\right|_{\varepsilon=0}=\max _{\substack{w \in W \\\|w\|_{2}=1}} w^{\top} Z w
$$

Proof Observe that

$$
\begin{aligned}
\|X+\varepsilon Z\| & \geq \max _{\|w\|_{2}=1, w \in W} w^{\top}(X+\varepsilon Z) w=\max _{\|w\|_{2}=1, w \in W} \underbrace{w^{\top} X w}_{=\lambda_{1}}+\varepsilon w^{\top} Z w \\
& =\lambda_{1}+\varepsilon \cdot \max _{\|w\|_{2}=1, w \in W} w^{T} Z w .
\end{aligned}
$$

It therefore suffices to show that $\|X+\varepsilon Z\|$ cannot exceed the lower bound by more than $o(\varepsilon)$.

Let $u$ be an arbitrary vector with $\|u\|_{2}=1$ and write $u=u_{1}+u_{2}$ with $u_{1} \in$ $W$ and $u_{2} \in W^{\perp}$, where the latter is the orthogonal complement of $W$. Clearly, $\left\|u_{1}\right\|_{2}^{2}+\left\|u_{2}\right\|_{2}^{2}=1$. Further let $\Delta:=\lambda_{1}-\lambda_{2}$ where $\lambda_{2}$ is the second largest Eigenvalue of $X$ and for readability let $\lambda_{1}(Z \uparrow W):=\max _{\|w\|_{2}=1, w \in W} w^{\top} Z w$. We estimate

$$
\begin{aligned}
u^{\top}(X+\varepsilon Z) u= & u_{1}^{\top} X u_{1}+u_{2}^{\top} X u_{2}+\varepsilon\left(u_{1}^{\top} Z u_{1}+u_{1}^{\top} Z u_{2}+u_{2}^{\top} Z u_{1}+u_{2}^{\top} Z u_{2}\right) \\
\leq & \lambda_{1}\left\|u_{1}\right\|_{2}^{2}+\lambda_{2}\left\|u_{2}\right\|_{2}^{2}+\varepsilon \lambda_{1}(Z \uparrow W)+3 \varepsilon\|Z\|\left\|u_{2}\right\|_{2} \\
= & \lambda_{1}+\varepsilon \lambda_{1}\left(Z\lceil W)+\left(3 \varepsilon\|Z\|-\Delta\left\|u_{2}\right\|_{2}\right)\left\|u_{2}\right\|_{2}\right. \\
= & \lambda_{1}+\epsilon \lambda_{1}\left(Z\lceil W)-\left(\sqrt{\Delta}\left\|u_{2}\right\|_{2}-3 \varepsilon\|Z\| / \sqrt{4 \Delta}\right)^{2}\right. \\
& +9 \varepsilon^{2}\|Z\|^{2} /(4 \Delta) \leq \lambda_{1}+\epsilon \lambda_{1}(Z \uparrow W)+9 \varepsilon^{2}\|Z\|^{2} /(4 \Delta),
\end{aligned}
$$

which finishes the proof.

We record the following corollary of Lemma 1 for later use. Recall that for a square matrix $X$, its exponential is given by

$$
e^{X}=\sum_{k=0}^{\infty} \frac{1}{k !} X^{k}=I+X+\frac{1}{2} X^{2}+\cdots
$$

Corollary 1 Let $r$ be a positive integer, $X \in \mathbb{S}_{+}^{r}$ be a non-zero positive semidefinite matrices. Let $\lambda_{1}=\|X\|$ and $W$ denote the $\lambda_{1}$-eigenspace of $X$. Then for $Z \in \mathbb{R}^{r \times r}$ symmetric, 


$$
\left.\frac{d_{+}}{d \varepsilon}\left\|e^{\varepsilon Z} X e^{\varepsilon Z}\right\|\right|_{\varepsilon=0}=2 \lambda_{1} \max _{\substack{w \in W \\\|w\|_{2}=1}} w^{\top} Z w
$$

Proof Let us write $e^{\varepsilon Z}=\sum_{k=0}^{\infty} \frac{\varepsilon^{k} Z^{k}}{k !}=I+\varepsilon Z+\varepsilon^{2} R_{\varepsilon}$, where $R_{\varepsilon}=\sum_{k=2}^{\infty} \frac{\varepsilon^{k-2} Z^{k}}{k !}$. For $\varepsilon<1 /(2\|Z\|)$, by the triangle inequality

$$
\left\|R_{\varepsilon}\right\| \leq \sum_{k=2}^{\infty} \frac{\varepsilon^{k-2}\|Z\|^{k}}{k !} \leq \frac{\|Z\|^{2}}{2} \sum_{k=0}^{\infty}(\varepsilon\|Z\|)^{k}=\frac{\|Z\|^{2}}{2(1-\varepsilon\|Z\|)} \leq\|Z\|^{2}
$$

From here we see that

$$
\begin{aligned}
e^{\varepsilon Z} X e^{\varepsilon Z}= & \left(I+\varepsilon Z+\varepsilon^{2} R_{\varepsilon}\right) X\left(I+\varepsilon Z+\varepsilon^{2} R_{\varepsilon}\right)=X+\varepsilon(Z X+X Z) \\
& +\varepsilon^{2}\left(Z X R_{\varepsilon}+R_{\varepsilon} X Z+R_{\varepsilon} X R_{\varepsilon}\right)
\end{aligned}
$$

Let $R_{\varepsilon}^{\prime}=Z X R_{\varepsilon}+R_{\varepsilon} X Z+R_{\varepsilon} X R_{\varepsilon}$. Again by the triangle inequality, we have that

$$
\left\|R_{\varepsilon}^{\prime}\right\| \leq 2\|Z\|\|X\|\left\|R_{\varepsilon}\right\|+\left\|R_{\varepsilon}\right\|^{2}\|X\| \leq 2\|Z\|^{3}\|X\|+\|Z\|^{4}\|X\|=O(1),
$$

for $\varepsilon$ small enough. Therefore, we have that

$$
\begin{aligned}
\left\|e^{\varepsilon Z} X e^{\varepsilon Z}\right\| & =\left\|X+\varepsilon(X Z+Z X)+\varepsilon^{2} R_{\varepsilon}^{\prime}\right\|=\|X+\varepsilon(X Z+Z X)\| \pm O\left(\varepsilon^{2}\left\|R_{\varepsilon}^{\prime}\right\|\right) \\
& =\|X+\varepsilon(X Z+Z X)\| \pm O\left(\varepsilon^{2}\right) .
\end{aligned}
$$

Since $X Z+Z X$ is symmetric and $X \in \mathbb{S}_{+}^{r}$ and non-zero, by Lemma 1 we have that

$$
\begin{aligned}
\|X+\varepsilon(X Z+Z X)\| & =\lambda_{1}+\varepsilon\left(\max _{\substack{w \in W \\
\|w\|_{2}=1}} w^{\top}(X Z+Z X) w\right) \pm O\left(\varepsilon^{2}\right) \\
& =\lambda_{1}+\varepsilon \lambda_{1}\left(\max _{\substack{w \in W \\
\|w\|_{2}=1}} w^{\top}(Z+Z) w\right) \pm O\left(\varepsilon^{2}\right) \\
& =\lambda_{1}+2 \lambda_{1} \varepsilon\left(\max _{\substack{w \in W \\
\|w\|_{2}=1}} w^{\top} Z w\right) \pm O\left(\varepsilon^{2}\right)
\end{aligned}
$$

Putting it all together, we get that

$$
\left\|e^{\varepsilon Z} X e^{\varepsilon Z}\right\|=\|X+\varepsilon(X Z+Z X)\|+O\left(\varepsilon^{2}\right)=\lambda_{1}+2 \lambda_{1} \varepsilon\left(\max _{\substack{w \in W \\\|w\|_{2}=1}} w^{\top} Z w\right) \pm O\left(\varepsilon^{2}\right)
$$

as needed. 


\section{Rescaling semidefinite factorizations}

A crucial point will be the rescaling of a semidefinite factorization of a nonnegative matrix $M$. In the case of linear extended formulations an upper bound of $\Delta$ on the largest entry of a slack matrix $S$ implies the existence of a minimal nonnegative factorization $S=U V$ where the entries of $U, V$ are bounded by $\sqrt{\Delta}$. This ensures that the approximation of the extended formulation can be captured by means of a polynomial-size (in $\Delta$ ) grid. In the linear case, we note that any factorization $S=$ $U V$ can be rescaled by a nonnegative diagonal matrix $D$ where $S=(U D)\left(D^{-1} U\right)$ and the factorization $\left(U D, D^{-1} V\right)$ has entries bounded by $\sqrt{\Delta}$. However, such a rescaling relies crucially on the fact that after independent nonnegative scalings of the coordinates a nonnegative vector remains nonnegative. However, in the PSD setting, it is not true that the PSD property is preserved after independent nonnegative scalings of the matrix entries. We circumvent this issue by showing that a restricted class of transformations, i.e., the symmetries of the semidefinite cone, suffice to rescale any PSD factorization such that the largest eigenvalue occurring in the factorization is bounded in terms of the maximum entry in $M$ and the rank of the factorization.

Theorem 6 (Rescaling semidefinite factorizations) Let $\Delta$ be a positive real number, $I, J$ be finite sets, $M \in[0, \Delta]^{I \times J}$ be a nonnegative matrix with a rank $r$ semidefinite factorization $(\mathbf{U}, \mathbf{V}), \mathbf{U}=\left(U_{i}\right)_{i \in I}, \mathbf{V}=\left(V^{j}\right)_{j \in J}$, satisfying $M_{i j}=\operatorname{Tr}\left[U_{i} V^{j}\right]$, $i \in I, j \in J$. Then there exists $A \in \mathbb{S}_{+}^{r}$ such that $A \mathbf{U} A=\left(A U_{i} A\right)_{i \in I}, A^{+} \mathbf{V} A^{+}=$ $\left(A^{+} V^{j} A^{+}\right)_{j \in J}$ is a semidefinite factorization of $M$ satisfying

$$
\begin{aligned}
\|A \mathbf{U} A\|_{\infty} & =\max _{i \in I}\left\|A U_{i} A\right\| \leq \sqrt{r \Delta} \\
\left\|A^{+} \mathbf{V} A^{+}\right\|_{\infty} & =\max _{j \in J}\left\|A^{+} V^{j} A^{+}\right\| \leq \sqrt{r \Delta} .
\end{aligned}
$$

Proof Let $\bar{U}=\sum_{i \in I} U_{i} /|I|, \bar{V}=\sum_{j \in J} V^{j} /|J|$. Let $W_{1}=\operatorname{Im}(\bar{U}), W_{2}=$ $\operatorname{Im}(\bar{V}), W=P_{W_{1}}\left(W_{2}\right)$ and $d=\operatorname{dim}(W)$. Let $O \in \mathbb{R}^{r \times d}$ denote an orthonormal basis matrix for $W$, that is $\operatorname{Im}(O)=W, O O^{\top}=P_{W}$, and $O^{\top} O=I_{d}$ (the $d \times d$ identity).

As a first step, we preprocess the factorization to make it full dimensional (i.e., by reducing the ambient dimension).

Claim $\left(O^{\top} \mathbf{U} O, O^{\top} \mathbf{V} O\right)$ is a semidefinite factorization of $M$. Furthermore, $O^{\top} \bar{U} O$ and $O^{\top} \bar{V} O$ are $d \times d$ nonsingular matrices.

Proof of Claim If $T \in \mathbb{S}_{+}^{r}$ then for any matrix $A \in \mathbb{R}^{r \times d}$, we have that $A^{\top} T A \in \mathbb{S}_{+}^{d}$. Hence $O^{\top} U_{i} O, O^{\top} V^{j} O \in \mathbb{S}_{+}^{d}$, for all $i \in I, j \in J$. To show that the new matrices factorize $M$, it suffices to show that $M_{i j}=\operatorname{Tr}\left[O^{\top} U_{i} O O^{\top} V^{j} O\right]$ for all $i \in I, j \in J$. We examine spectral decompositions of $U_{i}$ and $V_{j}$,

$$
U_{i}=\sum_{k=1}^{r} \lambda_{k} u_{k} u_{k}^{\top} \quad \text { and } \quad V^{j}=\sum_{k=1}^{r} \gamma_{k} v_{k} v_{k}^{\top} .
$$


For $k \in[r]$, we have that $u_{k} \in \operatorname{Im}\left(U_{i}\right) \subseteq \sum_{i \in I} \operatorname{Im}\left(U_{i}\right)=\operatorname{Im}(\bar{U})=W_{1}$. Similarly for $l \in[r], v_{l} \in \operatorname{Im}\left(V^{j}\right) \subseteq \operatorname{Im}(\bar{V})=W_{2}$. Given the previous containment, remembering that $P_{W_{1}}\left(W_{2}\right)=W$, for $k, l \in[r]$ we have that

$$
\left\langle u_{k}, v_{l}\right\rangle=\left\langle P_{W_{1}} u_{k}, v_{l}\right\rangle=\left\langle u_{k}, P_{W_{1}} v_{l}\right\rangle=\left\langle u_{k}, P_{W} v_{l}\right\rangle=\left\langle P_{W} u_{k}, P_{W} v_{l}\right\rangle=\left\langle O^{\top} u_{k}, O^{\top} v_{l}\right\rangle,
$$

since $O$ is an orthonormal basis matrix for $W$. The trace inner product can now be analyzed as follows

$$
\begin{aligned}
\operatorname{Tr}\left[U_{i} V^{j}\right] & =\sum_{1 \leq k, l \leq r} \lambda_{k} \gamma_{l}\left\langle u_{k}, v_{l}\right\rangle^{2}=\sum_{1 \leq k, l \leq r} \lambda_{k} \gamma_{l}\left\langle O^{\top} u_{k}, O^{\top} v_{l}\right\rangle^{2} \\
& =\operatorname{Tr}\left[\left(O^{\top} U_{i} O\right)\left(O^{\top} V^{j} O\right)\right]
\end{aligned}
$$

Hence $\left(O^{\top} \mathbf{U} O, O^{\top} \mathbf{V} O\right)$ is a semidefinite factorization of $M$ as needed.

For the furthermore, we must show that the matrices $O^{\top} \bar{U} O$ and $O^{\top} \bar{V} O$ have trivial kernels. By construction $\operatorname{Ker}(\bar{U})=W_{1}^{\perp}, \operatorname{Ker}(\bar{V})=W_{2}^{\perp}$, and

$$
W=P_{W_{1}}\left(W_{2}\right)=\left(W_{2}+W_{1}^{\perp}\right) \cap W_{1} .
$$

From here, we have that

$$
\begin{aligned}
\operatorname{dim}\left(\operatorname{Ker}\left(O^{\top} \bar{U} O\right)\right) & =\operatorname{dim}(\operatorname{Ker}(\bar{U} O))=\operatorname{dim}\left(W_{1}^{\perp} \cap W\right) \leq \operatorname{dim}\left(W_{1}^{\perp} \cap W_{1}\right) \\
& =\operatorname{dim}(\{0\})=0 .
\end{aligned}
$$

Next, we have that

$$
\begin{aligned}
\operatorname{dim}\left(\operatorname{Ker}\left(O^{\top} \bar{V} O\right)\right) & =\operatorname{dim}(\operatorname{Ker}(\bar{V} O))=\operatorname{dim}\left(W_{2}^{\perp} \cap W\right) \\
& =\operatorname{dim}\left(\left(W_{2}^{\perp} \cap W_{1}\right) \cap\left(W_{2}+W_{1}^{\perp}\right)\right) \\
& =\operatorname{dim}\left(\left(W_{2}+W_{1}^{\perp}\right)^{\perp} \cap\left(W_{2}+W_{1}^{\perp}\right)\right)=\operatorname{dim}(\{0\})=0,
\end{aligned}
$$

as needed.

We will now examine factorizations of the form $\left(A O^{\top} \mathbf{U} O A, A^{-1} O^{\top} \mathbf{V} O A^{-1}\right)$, for $A \in \mathbb{S}_{+}^{d}$ nonsingular. To see that this yields a factorization, note that

$$
\begin{gathered}
\operatorname{Tr}\left[A O^{\top} U_{i} O A A^{-1} O^{\top} V^{j} O A^{-1}\right]=\operatorname{Tr}\left[A O^{\top} U_{i} O O^{\top} V^{j} O A^{-1}\right] \\
=\operatorname{Tr}\left[O^{\top} U_{i} O O^{\top} V^{j} O A^{-1} A\right]=\operatorname{Tr}\left[O^{\top} U_{i} O O^{\top} V^{j} O\right]=M_{i j}
\end{gathered}
$$

where the last inequality follows from the claim above. To prove the theorem, it suffices to construct a nonsingular matrix $A \in \mathbb{S}_{+}^{d}$ such that

$$
\left\|A O^{\top} \mathbf{U} O A\right\|_{\infty} \leq \sqrt{d \Delta} \text { and }\left\|A^{-1} O^{\top} \mathbf{V} O A^{-1}\right\|_{\infty} \leq \sqrt{d \Delta}
$$


Given such an $A$, we can recover the rescaling matrix claimed in the theorem using $O A O^{\top}$, where $\left(O A O^{\top}\right)^{+}=O A^{-1} O^{\top}$. It is easy to check that this lifting is valid and preserves the maximum eigenvalues of the factorization matrices.

Given the above reduction, we may now assume that $d=r$ and that $\bar{U}, \bar{V}$ are nonsingular. We define the following potential function over factorizations,

$$
\Phi_{M}(\mathbf{U}, \mathbf{V})=\|\mathbf{U}\|_{\infty} \cdot\|\mathbf{V}\|_{\infty}
$$

We now examine the optimization problem

$$
\inf _{\substack{A \in \mathbb{S}_{+}^{r} \\ A \text { nonsingular }}} \Phi_{M}\left(A \mathbf{U} A, A^{-1} \mathbf{V} A^{-1}\right)
$$

For any nonsingular $T \in \mathbb{R}^{r \times r},\left(T \mathbf{U} T^{\top}, T^{-\top} \mathbf{V} T^{-1}\right)$ is a valid PSD factorization of $M$. Without loss of generality we can require $T$ to be PSD as above, since $T$ can be always be expressed as $T=O A$, where $O$ is orthogonal and $A \in \mathbb{S}_{+}^{r}$. Here it is easy to check that substituting $A$ for $T$ does not change the $\Phi_{M}$ value of the factorization.

Recall that the goal is to construct a nonsingular $A \in \mathbb{S}_{+}^{r}$ such that

$$
\|A \mathbf{U} A\|_{\infty} \leq \sqrt{r \Delta} \text { and }\left\|A^{-1} \mathbf{V} A^{-1}\right\|_{\infty} \leq \sqrt{r \Delta}
$$

For any scalar $s>0$, we see that

$$
\|s A \mathbf{U} s A\|_{\infty}=s^{2}\|A \mathbf{U} A\|_{\infty} \quad \text { and } \quad\left\|(s A)^{-1} \mathbf{V}(s A)^{-1}\right\|_{\infty}=\left\|A^{-1} \mathbf{V} A^{-1}\right\|_{\infty} / s^{2} .
$$

Given this, if $\Phi_{M}\left(A \mathbf{U} A, A^{-1} \mathbf{V} A^{-1}\right) \leq \mu^{2}$ then setting

$$
s=\Phi_{M}\left(A \mathbf{U} A, A^{-1} \mathbf{V} A^{-1}\right)^{1 / 4} /\|A \mathbf{U} A\|_{\infty}^{1 / 2},
$$

we get that

$$
\|s A \mathbf{U} s A\|_{\infty}=\left\|(s A)^{-1} \mathbf{V}(s A)^{-1}\right\|_{\infty}=\Phi_{M}\left(A \mathbf{U} A, A^{-1} \mathbf{V} A^{-1}\right)^{1 / 2} \leq \mu
$$

Hence it suffices to show that the infimum value for (1) is less than or equal to $r \Delta$. We claim that this infimum is attained. Since the objective functions is clearly continuous in $A$, it suffices to show that the infimum can be taken over a compact subset of $\mathbb{S}_{+}^{r}$. Let $\tau=\Phi_{M}(\mathbf{U}, \mathbf{V})$, and $\sigma>0$ be the largest value such that $\bar{U} \succeq \sigma I_{r}, \bar{V} \succeq \sigma I_{r}$. Note that $\sigma>0$ exists since $\bar{U}, \bar{V}$ are nonsingular $r \times r$ PSD matrices.

Claim Let $A \in \mathbb{S}_{+}^{r}$ nonsingular. If $\Phi_{M}\left(A \mathbf{U} A, A^{-1} \mathbf{V} A^{-1}\right) \leq \tau$, then there exists $s>0$ such that $I_{r} \preceq s A \preceq\left(\tau / \sigma^{2}\right) I_{r}$. 
Proof of Claim We examine the spectral decomposition of $A=\sum_{i=1}^{r} \lambda_{i} v_{i} v_{i}^{\top}$, where $v_{1}, \ldots, v_{r}$ form an orthornomal basis of $\mathbb{R}^{r}$ and $\lambda_{1} \geq \ldots \geq \lambda_{r} \geq 0$. Note that $A^{-1}=\sum_{i=1}^{r} \lambda_{i}^{-1} v_{i} v_{i}^{\top}$. Here $\lambda_{r}>0$ since $A$ is nonsingular. Since multiplying $A$ by a positive scalar does not change the potential $\Phi_{M}$, we may rescale $A$ such that $\lambda_{r}=1$. Since $\lambda_{r} I_{r} \preceq A \preceq \lambda_{1} I_{r}$, and $\lambda_{r}=1$, we must now show that $\lambda_{1} \leq \tau / \sigma^{2}$.

We lower bound $\Phi(A)$ in terms of $\lambda_{1}$. Firstly, note that

$$
\begin{aligned}
\|A \mathbf{U} A\|_{\infty} & =\max _{i \in I}\left\|A U_{i} A\right\| \geq \max _{i \in I} v_{1}^{\top} A U_{i} A v_{1} \geq \lambda_{1} \max _{i \in I} v_{1}^{\top} U_{i} v_{1} \\
& \geq \lambda_{1} \frac{1}{|I|} \sum_{i \in I} v_{1}^{\top} U_{i} v_{1}=\lambda_{1} v_{1}^{\top} \bar{U} v_{1} \geq \sigma \lambda_{1} .
\end{aligned}
$$

Next, we have that

$$
\begin{aligned}
\left\|A^{-1} \mathbf{V} A^{-1}\right\|_{\infty} & =\max _{j \in J}\left\|A^{-1} V^{j} A^{-1}\right\| \geq \max _{j \in J} v_{r}^{\top} A^{-1} V^{j} A^{-1} v_{r}=\lambda_{r}^{-1} \max _{j \in J} v_{r}^{\top} V^{j} v_{r} \\
& =\max _{j \in J} v_{r}^{\top} V^{j} v_{r} \geq \frac{1}{|J|} \sum_{j \in J} v_{r}^{\top} V^{j} v_{r}=v_{r}^{\top} \bar{V} v_{r} \geq \sigma .
\end{aligned}
$$

Therefore

$$
\tau \geq\|A \mathbf{U} A\|_{\infty}\left\|A^{-1} \mathbf{V} A^{-1}\right\|_{\infty} \geq \lambda_{1} \sigma^{2} \Rightarrow \lambda_{1} \leq \tau / \sigma^{2}
$$

as needed.

From the above claim, and our assumption that $\Phi_{M}(\mathbf{U}, \mathbf{V})=\tau$, we get that

$$
\inf _{\substack{A \in \mathbb{S}_{+}^{r} \\ A \text { nonsingular }}} \Phi_{M}\left(A \mathbf{U} A, A^{-1} \mathbf{V} A^{-1}\right)=\inf _{\substack{A \in \mathbb{S}_{+}^{r} \\ I_{r} \preceq A \preceq\left(\tau / \sigma^{2}\right) I_{r}}} \Phi_{M}\left(A \mathbf{U} A, A^{-1} \mathbf{V} A^{-1}\right)
$$

Since the infimum on the right hand side is taken on a compact set, the infimum is attained as claimed. Let $\mu^{2}$ denote the infimum value. Letting $(\widetilde{\mathbf{U}}, \widetilde{\mathbf{V}})=$ ( $A \mathbf{U} A, A^{-1} \mathbf{V} A^{-1}$ ), for the appropriate matrix $A \in \mathbb{S}_{+}^{r}$, we can assume

$$
\|\widetilde{\mathbf{U}}\|_{\infty}=\|\widetilde{\mathbf{V}}\|_{\infty}=\Phi_{M}(\tilde{\mathbf{U}}, \tilde{\mathbf{V}})^{1 / 2}=\mu
$$

We shall now analyze how $\Phi_{M}$ behaves under small perturbations of the minimizer $(\widetilde{\mathbf{U}}, \widetilde{\mathbf{V}})$. Our goal is to obtain a contradiction by assuming that $\mu^{2}>\Delta r+\tau$ for some $\tau>0$. To this end we bound the value of $\Phi_{M}$ at infinitesimal perturbations of the point $(\widetilde{\mathbf{U}}, \widetilde{\mathbf{V}})$. For a symmetric matrix $Z$ and parameter $\varepsilon>0$ the type of perturbations we consider are those defined by the invertible matrix $e^{-\varepsilon Z}$, which will take the role of the matrix $A$ above. Notice that if $Z$ is symmetric, then so is $e^{-\varepsilon Z}$. We show that there exists a matrix $Z$ such that for every $U \in\left\{\widetilde{U}_{i} \mid i \in I\right\}$ such that 
$\|U\|=\mu$, we have

$$
\left\|e^{-\varepsilon Z} U e^{-\varepsilon Z}\right\| \leq \mu-\frac{2 \mu}{r} \varepsilon+O\left(\varepsilon^{2}\right),
$$

while at the same time for every $V \in\left\{\widetilde{V}^{j} \mid j \in J\right\}$ such that $\|V\|=\mu$, we have

$$
\left\|e^{\varepsilon Z} V e^{\varepsilon Z}\right\| \leq \mu+\frac{2 \Delta}{\mu} \varepsilon+O\left(\varepsilon^{2}\right) \text {. }
$$

This implies that there is a point $\left(\mathbf{U}^{\prime}, \mathbf{V}^{\prime}\right)$ in the neighborhood of the minimizer $(\widetilde{\mathbf{U}}, \widetilde{\mathbf{V}})$ where

$$
\begin{aligned}
\Phi_{M}\left(\mathbf{U}^{\prime}, \mathbf{V}^{\prime}\right) & \leq\left(\mu-\frac{2 \mu}{r} \varepsilon+O\left(\varepsilon^{2}\right)\right) \cdot\left(\mu+\frac{2 \Delta}{\mu} \varepsilon+O\left(\varepsilon^{2}\right)\right) \\
& =\mu^{2}-2\left(\frac{\mu^{2}}{r}-\Delta\right) \varepsilon+O\left(\varepsilon^{2}\right) \\
& <\mu^{2}-\frac{2 \tau}{r} \varepsilon+O\left(\varepsilon^{2}\right),
\end{aligned}
$$

where the last inequality follows from our assumption that $\mu^{2}>\Delta r+\tau$. Thus, for small enough $\varepsilon>0$, we have $\Phi_{M}\left(\mathbf{U}^{\prime}, \mathbf{V}^{\prime}\right)<\mu^{2}$, a contradiction to the minimality of $\mu$. It suffices to consider the factorization matrices with the largest eigenvalues as small perturbations cannot change the eigenvalue structure. Hence, to prove the theorem we need to show the existence of such a matrix $Z$.

Let $\mathcal{Z} \subseteq S^{r-1}$ be a finite set of unit vectors such that every $z \in \mathcal{Z}$ is a $\mu$-eigenvector of at least one of the matrices $\widetilde{U}_{i}$ for $i \in I$. Let $p \in \mathbb{R}_{+}^{\mathcal{Z}}$ be a probability vector (i.e., $\sum_{z \in \mathcal{Z}} p(z)=1$ ) and define the symmetric matrix

$$
Z=\sum_{z \in \mathcal{Z}} p(z) z z^{\top}
$$

Claim Let $V \in\left\{\widetilde{V}^{j} \mid j \in J\right\}$ be one of the factorization matrices such that $\|V\|=\mu$. Then,

$$
\left.\frac{d_{+}}{d \varepsilon}\left\|e^{\varepsilon Z} V e^{\varepsilon Z}\right\|\right|_{\varepsilon=0} \leq \frac{2 \Delta}{\mu} .
$$

Proof of Claim Let $\mathcal{V} \subseteq S^{r-1}$ be the set of eigenvectors of $V$ that have eigenvalue $\mu$. Then, Corollary 1 gives

$$
\left.\frac{d_{+}}{d \varepsilon}\left\|e^{\varepsilon Z} V e^{\varepsilon Z}\right\|\right|_{\varepsilon=0}=2 \mu \max _{v \in \mathcal{V}} v^{\top} Z v=2 \mu \max _{v \in \mathcal{V}} \sum_{z \in \mathcal{Z}} p(z)\left(z^{\top} v\right)^{2}
$$

We show that for any $z \in \mathcal{Z}$ and $v \in \mathcal{V}$, we have $\left(z^{\top} v\right)^{2} \leq \Delta / \mu^{2}$. The claim then follows from (6) since $p$ is a probability vector. Let us fix vectors $z \in \mathcal{Z}$ and $v \in \mathcal{V}$ 
and let $U \in\left\{\widetilde{U}_{i} \mid i \in I\right\}$ be a factorization matrix such that $z$ is a $\mu$-eigenvector of $U$. Recall that the matrices $U$ and $V$ are part of a semidefinite factorization of the matrix $M$ and that we assumed the entries of $M$ to have value at most $\Delta$. Hence, $\operatorname{Tr}\left[U^{\top} V\right] \leq \Delta$. We now argue that $\mu^{2}\left(z^{\top} v\right) \leq \operatorname{Tr}\left[U^{\top} V\right]$. Let $U=\sum_{k \in[r]} \lambda_{k} u_{k} u_{k}^{\top}$ and $V=\sum_{\ell \in[r]} \gamma_{\ell} v_{\ell} v_{\ell}^{\top}$ be spectral decompositions of $U$ and $V$, respectively, such that $u_{1}=z$ and $v_{1}=v$. The $\lambda_{k}$ and $\gamma_{\ell}$ are nonnegative (as $U, V$ are PSD) and $\lambda_{1}=$ $\gamma_{1}=\mu$. Hence, expanding the trace inner product

$$
\operatorname{Tr}\left[U^{\top} V\right]=\sum_{k, \ell \in[r]} \lambda_{k} \gamma_{\ell}\left(u_{k}^{\top} v_{\ell}\right)^{2}
$$

we get that the terms on the right-hand side of (7) are nonnegative and that the sum in (7) is at least $\lambda_{1} \gamma_{1}\left(u_{1}^{\top} v_{1}\right)^{2}=\mu^{2}\left(z^{\top} v\right)^{2}$. Putting these observations together we conclude that $\mu^{2}\left(z^{\top} v\right)^{2} \leq \operatorname{Tr}\left[U^{\top} V\right] \leq \Delta$, which proves the claim.

Claim There exists a choice of unit vectors $\mathcal{Z}$ and probabilities $p$ such that the following holds. Let $I^{\prime}=\left\{i \in I \mid\left\|\widetilde{U}_{i}\right\|=\mu\right\}$. Then, for $Z$ as in (4) we have

$$
\left.\frac{d_{+}}{d \varepsilon}\left\|e^{-\varepsilon Z} \widetilde{U}_{i} e^{-\varepsilon Z}\right\|\right|_{\varepsilon=0} \leq-\frac{2 \mu}{r} \quad \forall i \in I^{\prime}
$$

Proof of Claim For every $i \in I^{\prime}$, let $\mathcal{U}_{i} \subseteq \mathbb{R}^{r}$ be the vector space spanned by the $\mu$ eigenvectors of $\widetilde{U}_{i}$. Define the convex set $\bar{K}=\operatorname{conv}\left(\bigcup_{i \in I^{\prime}}\left(\mathcal{U}_{i} \cap B_{2}^{r}\right)\right)$. Notice that $K$ is centrally symmetric. Let $k=\operatorname{dim}(K)$, and let $T \in \mathbb{R}^{r \times k}$ denote a linear transformation such that $E=T B_{2}^{k}$ is the smallest volume ellipsoid containing $K$. By John's Theorem, there exists a finite set $\mathcal{Z} \subseteq \operatorname{relbd}(K) \cap \operatorname{relbd}(E)$ and a probability vector $p \in \mathbb{R}_{+}^{\mathcal{Z}}$ such that

$$
Z=\sum_{z \in \mathcal{Z}} p(z) z z^{\top}=\frac{1}{k} T T^{\top}
$$

Notice that each $z \in \mathcal{Z}$ must be an extreme point of $K$ (as it is one for $E$ ) and the set of extreme points of $K$ is exactly $\bigcup_{i \in I^{\prime}}\left(\mathcal{U}_{i} \cap S^{r-1}\right)$. Hence, each $z \in \mathcal{Z}$ is a unit vector and at the same time a $\mu$-eigenvector of some $\widetilde{U}_{i}, i \in I^{\prime}$.

For $i \in I^{\prime}$, by Corollary 1 and (9) we have that

$$
\begin{aligned}
\left.\frac{d_{+}}{d \varepsilon}\left\|e^{-\varepsilon Z} \widetilde{U}_{i} e^{-\varepsilon Z}\right\|\right|_{\varepsilon=0} & =2 \mu \max \left\{u^{\top}(-Z) u \mid u \in \mathcal{U}_{i} \cap S^{r-1}\right\} \\
& =-2 \mu \min \left\{u^{\top} Z u \mid u \in \mathcal{U}_{i} \cap S^{r-1}\right\} \\
& =-\frac{2 \mu}{k} \min \left\{u^{\top} T T^{\top} u \mid u \in \mathcal{U}_{i} \cap S^{r-1}\right\} \\
& \leq-\frac{2 \mu}{r} \min \left\{\left\|T^{\top} u\right\|_{2}^{2} \mid u \in \mathcal{U}_{i} \cap S^{r-1}\right\} .
\end{aligned}
$$


Since $E \supseteq K \supseteq\left(\mathcal{U}_{i} \cap S^{r-1}\right)$, for any $u \in \mathcal{U}_{i} \cap S^{r-1}$, we have

$$
\left\|T^{\top} u\right\|_{2}=\sup _{x \in E} x^{\top} u \geq \sup _{y \in K} y^{\top} u \geq u^{\top} u=1 \text { as needed. }
$$

Notice that the first claim implies (3) and the second claim implies (2). Hence, our assumption $\mu^{2}>\Delta r+\tau$ contradicts that $\mu$ is the minimum value of $\Phi_{M}$.

\section{0/1 Polytopes with high semidefinite xc}

The lower bound estimation will crucially rely on the fact that any $0 / 1$ polytope in the $n$-dimensional unit cube can be written as a linear system of inequalities $A x \leq b$ with integral coefficients where the largest coefficient is bounded by $(\sqrt{n+1})^{n+1} \leq$ $2^{n \log (n)}$, see e.g., [18, Corollary 26]. Using Theorem 6 the proof follows along the lines of Rothvoß [13]; for simplicity and exposition we chose a compatible notation. We use different estimation however and we need to invoke Theorem 6 . In the following let $\mathbb{S}_{+}^{r}(\alpha)=\left\{X \in \mathbb{S}_{+}^{r} \mid\|X\| \leq \alpha\right\}$.

Lemma 2 (Rounding lemma) For a positive integer $n$ set $\Delta:=(n+1)^{(n+1) / 2}$. Let $\mathcal{X} \subseteq\{0,1\}^{n}$ be a nonempty set, let $r:=\operatorname{xc}_{S D P}(\operatorname{conv}(\mathcal{X}))$ and let $\delta \leq\left(16 r^{3}(n+\right.$ $\left.\left.r^{2}\right)\right)^{-1}$. Then, for every $i \in\left[n+r^{2}\right]$ there exist:

1. an integer vector $a_{i} \in \mathbb{Z}^{n}$ such that $\left\|a_{i}\right\|_{\infty} \leq \Delta$,

2. an integer $b_{i}$ such that $\left|b_{i}\right| \leq \Delta$,

3. a matrix $U_{i} \in \mathbb{S}_{+}^{r}(\sqrt{r \Delta})$ whose entries are integer multiples of $\delta / \Delta$ and have absolute value at most $8 r^{3 / 2} \Delta$, such that

$$
\begin{aligned}
\mathcal{X} & =\left\{x \in\{0,1\}^{n}\left|\exists Y \in \mathbb{S}_{+}^{r}(\sqrt{r \Delta}):\right| b_{i}-a_{i}^{\top} x-\left\langle Y, U_{i}\right\rangle \mid\right. \\
& \left.\leq \frac{1}{4\left(n+r^{2}\right)} \forall i \in\left[n+r^{2}\right]\right\} .
\end{aligned}
$$

Proof For some index set $I$ let $\mathcal{A}=\left(a_{i}, b_{i}\right)_{i \in I} \subseteq \mathbb{Z}^{n} \times \mathbb{Z}$ be a non-redundant description of conv $(\mathcal{X})$ (i.e., $|I|$ is minimal) such that for every $i \in I$, we have $\left\|a_{i}\right\|_{\infty} \leq \Delta$ and $\left|b_{i}\right| \leq \Delta$. Let $J$ be an index set for $\mathcal{X}=\left(x_{j}\right)_{j \in J}$ and let $S \in \mathbb{Z}_{\geq 0}^{I \times J}$ be the slack matrix of conv $(\mathcal{X})$ associated with the pair $(\mathcal{A}, \mathcal{X})$. The largest entry of the slack matrix is at most $\Delta$. By Yannakakis's Theorem (Theorem 4) there exists a semidefinite factorization $\left(U_{i}, V^{j}\right)_{(i, j) \in I \times J} \subseteq \mathbb{S}_{+}^{r} \times \mathbb{S}_{+}^{r}$ of $S$ such that

$$
\operatorname{conv}(\mathcal{X})=\left\{x \in \mathbb{R}^{n} \mid \exists Y \in \mathbb{S}_{+}^{r}: a_{i}^{\top} x+\left\langle U_{i}, Y\right\rangle=b_{i} \forall i \in I\right\}
$$

By Theorem 6 we may assume that $\left\|U_{i}\right\| \leq \sqrt{r \Delta}$ for every $i \in I$ and $\left\|V^{j}\right\| \leq \sqrt{r \Delta}$ for every $j \in J$. We will now pick a subsystem of maximum volume. For a linearly 
independent set of vectors $x_{1}, \ldots, x_{k} \in \mathbb{R}^{n}$, we let vol $\left(\left\{x_{1}, \ldots, x_{k}\right\}\right)$ denote the $k$ dimensional parallelepiped volume

$$
\operatorname{vol}\left(\sum_{i=1}^{k} a_{i} x_{i} \mid a_{1}, \ldots, a_{k} \in[0,1]\right)=\operatorname{det}\left(\left(x_{i}^{\top} x_{j}\right)_{i j}\right)^{\frac{1}{2}}
$$

If the vectors are dependent, then by convention the volume is zero. Let $\mathcal{W}=$ $\operatorname{span}\left\{\left(a_{i}, U_{i}\right) \mid i \in I\right\}$ and let $I^{\prime} \subseteq I$ be a subset of size $\left|I^{\prime}\right|=\operatorname{dim}(\mathcal{W})$ such that $\operatorname{vol}\left(\left\{\left(a_{i}, U_{i}\right) \mid i \in I^{\prime}\right\}\right)$ is maximized. Note that $\left|I^{\prime}\right| \leq n+r^{2}$.

For any positive semidefinite matrix $U \in \mathbb{S}_{+}^{r}$ with spectral decomposition

$$
U=\sum_{k \in[r]} \lambda_{k} u_{k} u_{k}^{\top}, \quad \text { we let } \quad \bar{U}=\sum_{k \in[r]} \bar{\lambda}_{k} \bar{u}_{k} \bar{u}_{k}^{\top}
$$

be the matrix where for every $k \in[r]$, the value of $\bar{\lambda}_{k}$ is the nearest integer multiple of $\delta / \Delta$ to $\lambda_{k}$ and $\bar{u}_{k}$ is the vector we get by rounding each of the entries of $u_{k}$ to the nearest integer multiple of $\delta / \Delta$. Since each $u_{k}$ is a unit vector, the matrices $u_{k} u_{k}^{\top}$ have entries in $[-1,1]$ and it follows that $U$ has entries in $r\|U\|[-1,1]$. Similarly, since each $\bar{u}_{k}$ has entries in $(1+\delta / \Delta)[-1,1]$ each of the matrices $\bar{u}_{k} \bar{u}_{k}^{\top}$ has entries in $(1+$ $\delta / \Delta)^{2}[-1,1]$, and it follows that $\bar{U}$ has entries in $r(\|U\|+\delta / \Delta)(1+\delta / \Delta)^{2}[-1,1]$. In particular, for every $i \in I^{\prime}$, the entries of $\bar{U}_{i}$ are bounded in absolute value by

$$
r\left(\left\|U_{i}\right\|+\delta / \Delta\right)(1+\delta / \Delta)^{2} \leq r(\sqrt{r \Delta}+\delta / \Delta)(1+\delta / \Delta)^{2} \leq 8 r^{3 / 2} \sqrt{\Delta}
$$

We use the following simple claim.

Claim Let $U$ and $\bar{U}$ be as above. Then, $\|\bar{U}-U\|_{2} \leq 4 \delta r^{2} / \sqrt{\Delta}$

Proof of Claim By the triangle inequality we have

$$
\begin{aligned}
\|\bar{U}-U\|_{F} & =\left\|\sum_{k \in[r]} \bar{\lambda}_{k} \bar{u}_{k} \bar{u}_{k}^{\top}-\lambda_{k} u_{k} u_{k}^{\top}\right\|_{F} \\
& \leq r \max _{k \in[r]}\left\|\bar{\lambda}_{k} \bar{u}_{k} \bar{u}_{k}^{\top}-\lambda_{k} u_{k} u_{k}^{\top}\right\|_{F} \\
& =r \max _{k \in[r]}\left\|\left(\bar{\lambda}_{k}-\lambda_{k}\right) \bar{u}_{k} \bar{u}_{k}^{\top}-\lambda_{k}\left(u_{k} u_{k}^{\top}-\bar{u}_{k} \bar{u}_{k}^{\top}\right)\right\|_{F} \\
& \leq r \max _{k \in[r]} \frac{\delta}{\Delta}\left\|\bar{u}_{k} \bar{u}_{k}^{\top}\right\|_{F}+\sqrt{r \Delta}\left\|u_{k} u_{k}^{\top}-\bar{u}_{k} \bar{u}_{k}^{\top}\right\|_{F} \\
& =r \max _{k \in[r]} \frac{\delta}{\Delta} \bar{u}_{k}^{\top} \bar{u}_{k}+\sqrt{r \Delta}\left\|\left(u_{k}-\bar{u}_{k}\right) u_{k}^{\top}-\bar{u}_{k}\left(\bar{u}_{k}^{\top}-u_{k}^{\top}\right)\right\|_{F} \\
& \leq r \max _{k \in[r]} \frac{\delta}{\Delta}\left(1+\frac{\delta}{\Delta} \sqrt{r}\right)^{2}+\sqrt{r \Delta}\left(\left\|u_{k}-\bar{u}_{k}\right\|_{F}+\left\|\bar{u}_{k}\right\|_{F}\left\|u_{k}-\bar{u}_{k}\right\|_{F}\right)
\end{aligned}
$$




$$
\begin{aligned}
& \leq r \frac{\delta}{\Delta}\left(1+\frac{\delta}{\Delta} \sqrt{r}\right)^{2}+r \sqrt{r \Delta}\left(\frac{\delta}{\Delta} \sqrt{r}+\left(1+\frac{\delta}{\Delta} \sqrt{r}\right) \frac{\delta}{\Delta} \sqrt{r}\right) \\
& \leq r \cdot 4 \delta r / \sqrt{\Delta}
\end{aligned}
$$

The claim now follows from the fact that $\delta \sqrt{r} / \Delta<1$.

Define the set

$$
\overline{\mathcal{X}}=\left\{x \in\{0,1\}^{n}\left|\exists Y \in \mathbb{S}_{+}^{r}(\sqrt{r \Delta}):\right| b_{i}-a_{i}^{\top} x-\left\langle\bar{U}_{i}, Y\right\rangle \mid \leq \frac{1}{4\left(n+r^{2}\right)} \forall i \in I^{\prime}\right\} .
$$

We claim that $\overline{\mathcal{X}}=\mathcal{X}$, which will complete the proof.

We will first show that $\mathcal{X} \subseteq \overline{\mathcal{X}}$. To this end, fix an index $j \in J$. By Theorem 4 we can pick $Y=V^{j} \in \mathbb{S}_{+}^{r}$ such that $a_{i}^{\top} x_{j}+\left\langle U_{i}, Y\right\rangle=b_{i}$ for every $i \in I^{\prime}$. Moreover, $\|Y\|=\left\|V^{j}\right\| \leq \sqrt{r \Delta}$. This implies that for every $i \in I^{\prime}$, we have

$$
\begin{aligned}
\left|b_{i}-a_{i}^{\top} x_{j}-\left\langle\bar{U}_{i}, Y\right\rangle\right| & =|\underbrace{b_{i}-a_{i}^{\top} x_{j}-\left\langle U_{i}, Y\right\rangle}_{0}+\left\langle\bar{U}_{i}-U_{i}, Y\right\rangle| \\
& \leq *\left\|\bar{U}_{i}-U_{i}\right\|_{F}\|Y\|_{F} \leq 4 \delta r^{3},
\end{aligned}
$$

where the second line follows from the Cauchy-Schwarz inequality, the above claim, and $\|Y\|_{F} \leq \sqrt{r}\|Y\| \leq r \sqrt{\Delta}$. Now, since $4 \delta r^{3} \leq 4 r^{3} /\left(16 r^{3}\left(n+r^{2}\right)\right)=1 /\left(4\left(n+r^{2}\right)\right)$ we conclude that $x_{j} \in \overline{\mathcal{X}}$ and hence $\mathcal{X} \subseteq \overline{\mathcal{X}}$.

It remains to show that $\overline{\mathcal{X}} \subseteq \mathcal{X}$. For this we show that whenever $x \in\{0,1\}^{n}$ is such that $x \notin \mathcal{X}$ it follows that $x \notin \overline{\mathcal{X}}$. To this end, fix an $x \in\{0,1\}^{n}$ such that $x \notin \mathcal{X}$. Clearly $x \notin \operatorname{conv}(\mathcal{X})$ and hence, there must be an $i^{*} \in I$ such that $a_{i^{*}}^{\top} x>b_{i^{*}}$. Since $x, a_{i^{*}}$ and $b_{i^{*}}$ are integral we must in fact have $a_{i^{*}}^{\top} x \geq b_{i^{*}}+1$. We express this violation in terms of the above selected subsystem corresponding to the set $I^{\prime}$.

There exist unique multipliers $v \in \mathbb{R}^{I^{\prime}}$ such that $\left(a_{i *}, U_{i^{*}}\right)=\sum_{i \in I^{\prime}} v_{i}\left(a_{i}, U_{i}\right)$. Observe that this implies that $\sum_{i \in I^{\prime}} v_{i} b_{i}=b_{i *}$; otherwise it would be impossible for $a_{i}^{\top} x+\left\langle U_{i}, Y\right\rangle=b_{i}$ to hold for every $i \in I$ and hence we would have $\mathcal{X}=\emptyset$ (which we assumed is not the case).

Using the fact that the chosen subsystem $I^{\prime}$ is volume maximizing and using Cramer's rule,

$$
\left|v_{i}\right|=\frac{\operatorname{vol}\left(\left\{\left(a_{t}, U_{t}\right) \mid t \in I^{\prime} \backslash\{i\} \cup\left\{i^{*}\right\}\right\}\right)}{\operatorname{vol}\left(\left\{\left(a_{t}, U_{t}\right) \mid t \in I^{\prime}\right\}\right)} \leq 1 .
$$

For any $Y \in \mathbb{S}_{+}^{r}(\sqrt{r \Delta})$ using $\left\langle U_{i^{*}}, Y\right\rangle \geq 0$ it follows thus

$$
\begin{aligned}
1 & \leq\left|a_{i^{*}}^{\top} x-b_{i^{*}}+\left\langle U_{i^{*}}, Y\right\rangle\right|=\left|\sum_{i \in I^{\prime}} v_{i}\left(a_{i}^{\top} x-b_{i}+\left\langle U_{i}, Y\right\rangle\right)\right| \\
& \leq \sum_{i \in I^{\prime}}\left|v_{i}\right|\left|a_{i}^{\top} x-b_{i}+\left\langle U_{i}, Y\right\rangle\right| \leq\left(n+r^{2}\right) \max _{i \in I^{\prime}}\left|a_{i}^{\top} x-b_{i}+\left\langle U_{i}, Y\right\rangle\right| .
\end{aligned}
$$


Using a similar estimation as above, for every $i \in I^{\prime}$, we have

$$
\begin{aligned}
\left|a_{i}^{\top} x-b_{i}+\left\langle U_{i}, Y\right\rangle\right| & =\left|a_{i}^{\top} x-b_{i}+\left\langle\bar{U}_{i}, Y\right\rangle+\left\langle U_{i}-\bar{U}_{i}, Y\right\rangle\right| \\
& \leq\left|a_{i}^{\top} x-b_{i}+\left\langle\bar{U}_{i}, Y\right\rangle\right|+\left|\left\langle U_{i}-\bar{U}_{i}, Y\right\rangle\right| \\
& \leq\left|a_{i}^{\top} x-b_{i}+\left\langle\bar{U}_{i}, Y\right\rangle\right|+\frac{1}{4\left(n+r^{2}\right)} .
\end{aligned}
$$

Combining this with $1 \leq\left(n+r^{2}\right) \max _{i \in I^{\prime}}\left|a_{i}^{\top} x-b_{i}+\left\langle U_{i}, Y\right\rangle\right|$ we obtain

$$
\frac{1}{2\left(n+r^{2}\right)} \leq \frac{1}{n+r^{2}}-\frac{1}{4\left(n+r^{2}\right)} \leq \max _{i \in I^{\prime}}\left|a_{i}^{\top} x-b_{i}+\left\langle\bar{U}_{i}, Y\right\rangle\right|
$$

and so $x \notin Y$.

Via padding with empty rows we can ensure that $\left|I^{\prime}\right|=n+r^{2}$ as claimed.

Using Lemma 2 we can establish the existence of 0/1 polytopes that do not admit any small semidefinite extended formulation following the proof of [13, Theorem 4].

Theorem 7 For any $n \in \mathbb{N}$ there exists $\mathcal{X} \subseteq\{0,1\}^{n}$ such that

$$
\operatorname{xc}_{S D P}(\operatorname{conv}(\mathcal{X}))=\Omega\left(\frac{2^{n / 4}}{(n \log n)^{1 / 4}}\right) .
$$

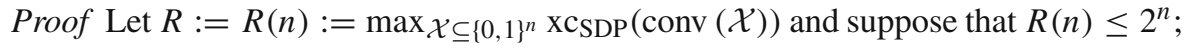
otherwise the statement is trivial. The construction of Lemma 2 induces an injective map from $\mathcal{X} \subseteq\{0,1\}^{n}$ to systems $\left(a_{i}, U_{i}, b_{i}\right)_{i \in\left[n+r^{2}\right]}$ as the set $\mathcal{X}$ can be reconstructed from the system. Also, adding zero rows and columns to $A, U$ and zero rows to $b$ does not affect this property. Thus without loss of generality we assume that $A$ is a $\left(n+R^{2}\right) \times n$ matrix, $U$ is a $\left(n+R^{2}\right) \times R^{2}$ matrix (using $\frac{R(R+1)}{2} \leq R^{2}$ ). Furthermore, by Lemma 2, every value in $U$ has absolute value at most $\Delta$ and can be chosen to be a multiple of $\left(16 R^{3}\left(n+R^{2}\right)\right)^{-1} \Delta^{-1}$. Thus each entry can take at most $3\left(16 R^{3}\left(n+R^{2}\right)\right) \Delta \cdot \Delta=\Delta^{2+o(1)}$ values, since $R \leq 2^{n}$ and $\Delta \geq n^{n / 2}$. Furthermore, the entries of $A, b$ are integral and have absolute value at most $\Delta$, and hence each entry can take at most $3 \Delta \leq \Delta^{2+o(1)}$ different values.

We shall now assume that $R \geq n$ (this will be justified by the lower bound on $R$ later). By injectivity we cannot have more sets than distinct systems, i.e.,

$$
2^{2^{n}}-1 \leq \Delta^{(2+o(1))\left(n+R^{2}+1\right)\left(n+R^{2}\right)}=\Delta^{(2+o(1)) R^{4}}=2^{(2+o(1)) n \log n R^{4}} .
$$

Hence for $n$ large enough, $R \geq \frac{2^{n / 4}}{(3 n \log n)^{1 / 4}}$ as needed.

\section{On the semidefinite xc of polygons}

In an analogous fashion to Fiorini et al. [7] we can use a slightly adapted version of Theorem 2 to show the existence of a polygon with $d$ integral vertices with semidefinite 
extension complexity $\Omega\left(\left(\frac{d}{\log d}\right)^{\frac{1}{4}}\right)$. For this we change Theorem 2 to work for arbitrary polytopes with bounded vertex coordinates; the proof is almost identical to Theorem 2 and follows with the analogous changes as in Fiorini et al. [7].

Lemma 3 (Generalized rounding lemma) Let $n, N \geq 2$ be a positive integer and set $\Delta:=((n+1) N)^{2 n}$. Let $\mathcal{V} \subseteq \mathbb{Z}^{n} \cap[-N, N]^{n}$ be a nonempty and convex independent set and $\mathcal{X}:=\operatorname{conv}(\mathcal{V}) \cap \mathbb{Z}^{n}$. With $r:=\operatorname{xc}_{S D P}(\operatorname{conv}(\mathcal{X}))$ and $\delta \leq\left(16 r^{3}\left(n+r^{2}\right)\right)^{-1}$, for every $i \in\left[n+r^{2}\right]$ there exist:

1. an integer vector $a_{i} \in \mathbb{Z}^{n}$ such that $\left\|a_{i}\right\|_{\infty} \leq \Delta$,

2. an integer $b_{i}$ such that $\left|b_{i}\right| \leq \Delta$,

3. a matrix $U_{i} \in \mathbb{S}_{+}^{r}(\sqrt{r \Delta})$ whose entries are integer multiples of $\delta / \Delta$ and have absolute value at most $8 r^{3 / 2} \Delta$, such that

$$
\mathcal{X}=\left\{x \in \mathbb{Z}^{n}\left|\exists Y \in \mathbb{S}_{+}^{r}(\sqrt{r \Delta}):\right| b_{i}-a_{i}^{\top} x-\left\langle Y, U_{i}\right\rangle \mid \leq \frac{1}{4\left(n+r^{2}\right)} \forall i \in\left[n+r^{2}\right]\right\} .
$$

Proof By, e.g., [10, Lemma D.4.1] it follows that $P$ has a non-redundant description with integral coefficients of largest absolute value of at most $((n+1) N)^{n}$. Thus the maximal entry occurring in the slack matrix is $((n+1) N)^{2 n}=\Delta$. The proof follows now with a similar argument as in Theorem 2.

We are ready to prove the existence of a polygon with $d$ vertices, with integral coefficients, so that its semidefinite extension complexity is $\Omega\left(\left(\frac{d}{\log d}\right)^{\frac{1}{4}}\right)$.

Theorem 8 (Integral polygon with high semidefinite $\mathrm{xc}$ ) For every $d \geq 3$, there exists ad-gon $P$ with vertices in $[2 d] \times\left[4 d^{2}\right]$ and $\operatorname{xc}_{S D P}(P)=\Omega\left(\left(\frac{d}{\log d}\right)^{\frac{1}{4}}\right)$.

Proof The proof is identical to the one is [7] except for adjusting parameters as follows. The set $Z:=\left\{\left(z, z^{2}\right) \mid z \in[2 d]\right\}$ is convex independent, thus every subset $X \subseteq Z$ of size $|X|=d$ yields a different convex $d$-gon. Let $R:=$ $\max \left\{\mathrm{xc}_{\mathrm{SDP}} \operatorname{conv}(X)|X \subseteq Z| X \mid,=d\right\}$.

As in the proof of Theorem 7, we need to count the number of systems (which the above set of polygons map to in an injective manner). Using $\Delta=\left(12 d^{2}\right)^{2}, n=$ 2, $N=4 d^{2}$ by Lemma 3 it follows easily that each entry in the system can take at most $c d^{14}$ different values. Without loss of generality, by padding with zeros, we assume that the system given by Lemma 3 has the following dimensions: the $A, b$ part from (1) and (2), where $A$ is formed by the rows $a_{i}$, is a $\left(3+R^{2}\right) \times 3$ matrix and $U$ from (3.), formed by the $U_{i}$ read as rows vectors, is a $\left(3+R^{2}\right) \times R^{2}$ matrix. We estimate

$$
2^{d} \leq\left(c d^{14}\right)^{\left(3+R^{2}\right)^{2}} \leq 2^{c^{\prime} \cdot R^{4} \cdot \log d}
$$

and hence $R \geq c^{\prime}\left(\frac{d}{\log d}\right)^{\frac{1}{4}}$ for some constant $c^{\prime}>0$ follows. 


\section{Final remarks}

Most of the questions and complexity theoretic considerations in Rothvoß [13] as well as the approximation theorem carry over immediately to our setting and the proofs follow similarly. For example, in analogy to [13, Theorem 6], an approximation theorem for $0 / 1$ polytopes can be derived showing that every semidefinite extended formulation for a $0 / 1$ polytope can be approximated arbitrarily well by one with coefficients of bounded size.

The following important problems remain open:

Problem 1 Does the CUT polytope have high semidefinite extension complexity. We highly suspect that the answer is in the affirmative, similar to the linear case. However the partial slack matrix analyzed in Fiorini et al. [6] to establish the lower bound for linear EFs has an efficient semidefinite factorization. In fact, it was precisely this fact that established the separation between semidefinite EFs and linear EFs in Braun et al. [3].

Problem 2 Is there an information theoretic framework for lower bounding semidefinite rank similar to the framework laid out in Braun et al. [4], Braun and Pokutta [2] for nonnegative rank?

Problem 3 As asked in Fiorini et al. [7], we can ask similarly for semidefinite EFs: is the provided lower bound for the semidefinite extension complexity of polygons tight?

Acknowledgments We are indebted to the anonymous referees for their remarks and the shortening of the proof of Lemma 1.

\section{References}

1. Ben-Tal, A., Nemirovski, A.: On polyhedral approximations of the second-order cone. Math. Oper. Res. 26, 193-205 (2001). doi:10.1287/moor.26.2.193.10561

2. Braun, G., Pokutta, S.: Common information and unique disjointness. In: IEEE 54th Annual Symposium on Foundations of Computer Science (FOCS 2013), pp. 688-697. Berkeley, California (2013)

3. Braun, G., Fiorini, S., Pokutta, S., Steurer, D.: Approximation limits of linear programs (Beyond Hierarchies). In: 53rd IEEE Symposium on Foundations of Computer Science (FOCS 2012), pp. 480-489 (2012). ISBN 978-1-4673-4383-1. doi:10.1109/FOCS.2012.10

4. Braverman, M., Moitra, A.: An information complexity approach to extended formulations. In: Proceedings of the 45th annual ACM symposium on Symposium on theory of computing, pp. 161-170. ACM (2013)

5. Faenza, Y., Fiorini, S., Grappe, R., Tiwary, H.R.: Extended formulations, nonnegative factorizations, and randomized communication protocols. In: Mahjoub, A., Markakis, V., Milis, I., Paschos, V. (eds.) Combinatorial Optimization, volume 7422 of Lecture Notes in Computer Science, pp. 129-140. Springer, Berlin (2012). ISBN 978-3-642-32146-7. doi:10.1007/978-3-642-32147-4_13.

6. Fiorini, S., Massar, S., Pokutta, S., Tiwary, H.R., de Wolf, R.: Linear vs semidefinite extended formulations exponential: separation and strong lower bounds. In: Proceedings of STOC (2012a)

7. Fiorini, S., Rothvoß, T., Tiwary, H.R.: Extended formulations for polygons. Discret. Comput. Geom. 48(3), 658-668 (2012b). ISSN 0179-5376. doi:10.1007/s00454-012-9421-9

8. Goemans, M.X.: Smallest compact formulation for the permutahedron. Math. Program. 1-7 (2014). doi:10.1007/s10107-014-0757-1

9. Gouveia, J., Parrilo, P.A., Thomas, R.: Lifts of convex sets and cone factorizations. Math. Oper. Res. 38(2), 248-264 (2011) 
10. Hindry, M., Silverman, J.H.: Diophantine Geometry: An Introduction. Springer, Berlin (2000)

11. John, F.: Extremum problems with inequalities as subsidiary conditions. In: Studies and Essays Presented to R. Courant on his 60th Birthday, pp. 187-204 (1948)

12. Katō, T.: Perturbation Theory for Linear Operators. Springer, Berlin (1995)

13. Rothvoß, T.: Some 0/1 polytopes need exponential size extended formulations. Math. Program. 142(12), 255-268 (2013). doi:10.1007/s10107-012-0574-3

14. Rothvoß, T.: The Matching Polytope has Exponential Extension Complexity. ArXiv e-prints (2013)

15. Shannon, C.E.: The synthesis of two-terminal switching circuits. Bell Syst. Tech. J. 25, 59-98 (1949)

16. Yannakakis, M.: Expressing combinatorial optimization problems by linear programs (extended abstract). In: Proceedings of the STOC 1988, pp. 223-228 (1988)

17. Yannakakis, M.: Expressing combinatorial optimization problems by linear programs. J. Comput. Syst. Sci. 43(3), 441-466 (1991). doi:10.1016/0022-0000(91)90024-Y

18. Ziegler, G.M.: Lectures on 0/1-Polytopes. In: Kalai, G., Ziegler, G.M. (eds.) Polytopes-Combinatorics and Computation, Volume 29 of DMV Seminar, pp. 1-41. Birkhäuser Basel (2000). ISBN 978-3-76436351-2. doi:10.1007/978-3-0348-8438-9_1 